\title{
Single fibre EMG studies in chronic fatigue syndrome: a reappraisal
}

\author{
Leslie Roberts, Edward Byrne
}

\begin{abstract}
Single fibre EMG studies were carried out on the right extensor digitorum communis muscle in $\mathbf{3 0}$ subjects with chronic fatigue syndrome and in 30 age and sex matched controls. Abnormal jitter was seen in five patients with chronic fatigue syndrome. Slight but significant differences between the mean consecutive differences in the remainder of the chronic fatigue subjects and the control subjects were recorded. Overall the differences were so minor that it seems unlikely that a disturbance of neuromuscular function as reflected by jitter measurement has a pathogenetic role. It is suggested that the increased jitter seen may be explained by the effects of the variability of motor unit firing rates on the myogenic component of the jitter.
\end{abstract}

(F Neurol Neurosurg Psychiatry 1994;57:375-376)

Chronic fatigue, often with muscle pain, is a prominent complaint in western countries and often a definable physical or psychiatric basis cannot be established. Idiopathic chronic fatigue syndrome has been defined by a Centre for Disease Control (CDC) committee $^{1}$ as disabling chronic fatigue of over six months' duration in the absence of a definable physical or psychiatric basis and a number of minor criteria are also required to establish the diagnosis. A great deal has been published about chronic fatigue syndrome in the immunological, ${ }^{2}$ virological, ${ }^{3}$ and psychiatric fields ${ }^{4}$ but its aetiology is still poorly understood. The suggestion that abnormal muscle metabolism may be at fault ${ }^{5}$ is not supported by other studies ${ }^{67}$ and muscle exercise performance ${ }^{8-10}$ is normal. A finding of abnormal single fibre jitter in $75 \%$ of cases in one study is perhaps the only remaining suggestion of a peripheral neuromuscular abnormality in this syndrome. ${ }^{11}$ The purpose of the present study was to re-examine this question in a cohort of patients with chronic fatigue syndrome with a carefully selected control group.

\section{Methods}

Thirty patients were admitted to the study, all of whom fitted CDC criteria for diagnosis.
All patients had moderate or severe fatigue and $92 \%$ had moderate or severe myalgia. All had symptoms for over 6 months and 54\% had symptoms for over 5 years. Although a history of antecedent infection was given in $40 \%$ of patients, serological confirmation was usually not available.

After informed consent was obtained, single fibre jitter studies were performed in the right extensor digitorum communis muscle. A standard Medelec SFEMG needle was used with a recording surface of $25 \mu \mathrm{m}$ diameter. Recordings were made with a Medelec MS6 electromyograph. The traces were digitised and transferred on line by a Medelec CI6 interface to an Epson PC (IBM compatible) computer for subsequent analysis. Eighteen to 20 recordings of each pair were acquired and stored in this way. At least 10 potential pairs were analysed. The recording was made with voluntary activation of the muscle, with the subject maintaining a slight, steady contraction.

The interpotential interval (IPI) and mean consecutive differences (MCD) were calculated. Thirty age and sex matched control subjects were studied in parallel. The mean age of the patient group was 39 (range 21 to 58) years and the mean age of the control group was 34 (range 21 to 55) years.

\section{Results}

The mean MCD of the controls was $25 \cdot 7$ (SD $4 \cdot 3$ ) $\mu \mathrm{s}$. The jitter was considered abnormal if it was greater than $3 \mathrm{SD}$ above the control mean, or if more than two pairs had jitter in excess of $55 \mu \mathrm{s}$ or if blocking was seen. Using these criteria five subjects with chronic fatigue syndrome had abnormal jitter. Blocking was seen in only two patients. One of these had $5 \%$ blocking in only $1 / 10$ pairs studied and the mean jitter was increased $(50.3 \mu \mathrm{s})$. The other had $5 \%$ blocking in $2 / 10$ pairs and the mean jitter was $49 \cdot 3 \mu \mathrm{s}$. Three other subjects with chronic fatigue syndrome had abnormal jitter (each with $2 / 10$ pairs greater than $55 \mu \mathrm{s}$, the mean jitter being 33.3 , 39.2 , and $43.0 \mu \mathrm{s}$ ). The cases with abnormal results had no clinical distinguishing features from other cases. The mean MCD of these five patients was 43.0 (SD $7 \cdot 1) \mu \mathrm{s}$. The mean MCD of the remaining 25 subjects with chronic fatigue syndrome was $27 \cdot 8$ (SD 
5.0) $\mu \mathrm{s}$. As expected the patients with abnormal results were significantly different from both the control group ( $p<0.001$, Student's $t$ test) and the remaining 25 patients ( $<<$ 0.001 , Student's $t$ test). There was also a very small but significant difference between the control group and the 25 patients with normal results ( $p<0.05$, Student's $t$ test). The MCD values did not correlate with the duration of illness, different clinical phenotypes, or history of an infectious disease at the onset. The interpotential intervals were recorded but are not reported here. They were all less than $2.5 \mathrm{~ms}$ and there was no significant difference between the control or either patient groups.

\section{Discussion}

This study has shown a small subgroup of patients with chronic fatigue syndrome who have abnormal single fibre jitter without any other evidence of motor unit abnormality. All of our patients with abnormal jitter had variable innervation rates associated with abnormal recruitment of motor units, which is a common finding in chronic fatigue syndromes. This finding was also common among the other patients. There was a slightly higher jitter in the patient $v$ the control group. The amount of this difference is very small and not clinically important. Fatigue due to neuromuscular transmission defects depends upon the presence of blocking and this was not present in most patients.

The reasons for the discrepancy between our findings and those of Jamal and Hansen, ${ }^{11}$ who found $75 \%$ of their patients had abnormal studies, is not clear. Comparison of data of this type with an age and sex matched control group, as in this study, rather than with standard laboratory reference ranges, is clearly essential, however.

Variability in jitter is usually due largely to neuromuscular transmission at the motor end plates. Variability of conduction in the terminal axon branches or variability of muscle fibre propagation may also contribute significantly to jitter. ${ }^{12}$

Trontelj et $a l^{13}$ have shown that the contribution to jitter from muscle fibre propagation (myogenic jitter) is normally small and with more or less constant rates of stimulation of axons, is less than $5 \mu \mathrm{s}$. Increased myogenic jitter can potentially be produced, however, by long mean interpotential intervals and with erratic discharge rates. ${ }^{13}$ In this study all interpotential intervals recorded were less than $2.5 \mathrm{~ms}$ and therefore variability in the interpotential intervals is not likely to be an important factor.

Erratic rates of stimulation of a muscle fibre will under normal conditions significantly change the velocity of propagation and this variation does occur at physiological rates of stimulation. Abnormalities of recruitment may therefore be sufficient to contribute to increased myogenic jitter; this may explain the abnormal studies reported here and also the slightly higher jitter seen in the chronic fatigue syndrome group. Also, in this study, calculations were made on a small number of motor unit discharges. This may have resulted in artefactually increasing the effects of erratic recruitment on the myogenic component of the jitter.

Direct stimulation of muscle fibres and stimulated SFEMG studies would eliminate the contribution of the effects of variation in rates of transmission in the axons and across the neuromuscular synapses and may help localise the site of origin of the abnormal jitter. The cause of the blocking seen in two patients is not clear. This was, however, only a minor finding present in very few pairs and it is probably of no clinical importance.

\section{Conclusion}

In this study only five out of 30 patients with chronic fatigue syndrome had an abnormal jitter and the mean of the jitter in the remaining patients was only slightly higher than in the controls. These abnormalities are considerably less than in a previously reported study. It is suggested that the increased jitter may be explained by variable rates of innervation producing an unusually large component of myogenic jitter rather than being related to primary effects of the disorder at the neuromuscular junction, terminal axon, or muscle membrane. The low incidence of abnormal studies and the minor abnormalities seen suggest that the increased jitter is not due to an important pathogenetic factor in chronic fatigue syndrome. In our hands, SFEMG is not a useful test in chronic fatigue syndrome, other than to exclude other conditions such as myasthenia gravis.

We thank St Vincent's Hospital for allowing us to study these patients and Dr Richard Kirsner for his statistical advice. The results of this study were presented in part at the VIIth International Congress on Neuromuscular Disease, Munich, West Germany, September 1990.

1 Holmes GP, Kaplan SE, Gantz NM, et al. Chronic fatigue syndrome: a working definition. Ann Intern Med 1988 syndrome:

2 Lloyd AR, Wakefield D, Broughton CR, Dwyer JM, Immunological abnormalities in the chronic fatigue syndrome. Med f Aust 1989;151:122-4.

3 Tobi M, Morag A, Ravid Z, et al. Prolonged atypical illnesses associated with serological evidence of persistent Epstein Barr virus infection. Lancet 1982;i:61-4.

4 Manu P, Matthews DA, Lane TS. The mental health of patients with a chief complaint of chronic fatigue. Arch Intern Med 1988;148:2213-7.

5 Arnold DL, Bure PS, Radda GK, Styles P, Taylor DJ. Excessive intracellular acidosis of skeletal muscle on exercise in a patient with post-viral/exhaustion fatigue syndrome. Lancet 1984;i:1367-9.

6 Byrne E, Trounce I. Chronic fatigue and myalgia syndrome. Mitochondrial and glycolytic studies in skeletal muscle. ₹ Neurol Neurosurg Psychiatry 1987;50:743-6.

7 Miller RG, Kostov K, Okechukwu C, Baker A, Weine MW. Correlated physiological and ${ }^{31} \mathrm{P}$ NMR spectroscopy studies of patients with chronic fatigue syndrome. scopy studies of patients with

8 Lloyd AR, Gandevia SC, Hales JP. Muscle performance, voluntary activation, twitch properties and perceived effort in normal subjects and patients with chronic fatigue syndrome. Brain 1991;114:85-98.

9 Lloyd AR, Hales JP, Gandevia SC. Muscle strength endurance and recovery in the post infectious fatigue syndrome. $f$ Neurol Neurosurg Psychiatry 1988;51: 1316-22.

10 Stokes MJ, Cooper M, Edwards R. Normal muscle strength and fatiguability in patients with effort syndrome. $B M \mathcal{F}$ 1988;297:1014-7.

11 Jamal JA, Hansen S. Electrophysiological studies in post viral fatigue syndrome. $\mathcal{F}$ Neurol Neurosurg Psychiatry 1988;48:691-4.

12 Stålberg E, Trontelj JV. In: Single fibre electromyography. Old Woking, Surrey: Mirvalle Press, 1979;1-244.

13 Trontelj JV, Stålberg E, Mihelin M. Jitter in the muscle fibre. F Neurol Neurosurg Psychiatry 1990;53:49-54. 